\title{
A Avaliação da Atenção Primária: um olhar preliminar através do PCATool em Manaus (AMAZONAS)
}

Lenice Ypiranga Benevides de Araújo Vieira Sá

\section{RESUMO}

Motivação: Este artigo tem por objetivo avaliar os atributos da atenção primária à saúde sob a perspectiva dos usuários do sistema de saúde pública no município de Manaus (AM).

Método: Realizou-se um estudo transversal, descritivo com uma população de 395 usuários cadastrados e atendidos pelas equipes da Estratégia Saúde da Família nas unidades básicas de saúde, os quais responderam com o uso do instrumento PCAToolBrasil (versão adultos) e a um questionário sociodemográfico.

Resultados: Os atributos da acessibilidade, integralidade, orientação comunitária obtiveram médias de escores baixos. O Escore Geral, mensurado pela média do Escore Essencial e do Escore Derivado, superou o ponte de corte de 6,6. A média do Escore Essencial foi de 7,16.

Conclusões: Os serviços e ações de saúde em Manaus de maneira geral possuem alto grau de orientação à Atenção Primária à Saúde, embora dois atributos essenciais e um atributo derivado possuam indicadores que necessitam ser melhorados.

Revista da Rede APS 2019 Publicada em: 24/07/2019

DOI:10.14295/aps.v1i2.27 Lenice Ypiranga Benevides de Araújo Vieira Sá (Departamento de Economia, Faculdade de Estudos Sociais, Universidade Federal do Amazonas, Brasil);

Correspondência para: Lenice Ypiranga Benevides de Araújo Vieira Sá, lenicebenevides@gmail.c om

Palavras-chave: Avaliação em Saúde; Avaliação da Atenção Primária; Atenção Primária à Saúde. 


\section{INTRODUÇÃO}

A Atenção Primária à Saúde (APS) configura aquele nível de atenção do sistema de saúde que funciona como porta de entrada para todas as necessidades de saúde das pessoas. Sendo assim, deve levar em conta os problemas mais comuns da comunidade, baseada na organização e racionalização do uso de todos os recursos (básicos e especializados) a fim de oferecer serviços de promoção, prevenção, cura e reabilitação (1).

O processo de construção do Sistema Único de Saúde em fins do século passado definiu uma nova perspectiva para o modelo de assistência pública à saúde no Brasil, ao incorporar na pauta de sua agenda política, diferentes estratégias de universalização da saúde entre as quais, a atenção primária à saúde. De início, esta última foi idealizada como um conjunto de ações seletivas e focalizadas orientadas ao combate à pobreza, na região Nordeste do País através da criação do Programa de Agentes Comunitários (PACS) em 1991. Posteriormente, ganhou importância como um modelo concebido para reestruturar os serviços de saúde de atenção primária no Brasil, em 1993, com a instituição do Programa Saúde da Família, depois denominado de Estratégia Saúde da Família (ESF) (2).

No final dos anos 90 observam-se esforços do Ministério da Saúde no sentido de propor a institucionalização da avaliação da atenção primária com vistas à superação dos seus limites organizacionais através de diversas ações/atividades, projetos e políticas, com o objetivo explícito de incorporar a avaliação no cotidiano de gestores e profissionais, partindo de uma visão abrangente dos processos avaliativos (3).
Dentre os instrumentos utilizados para avaliar a qualidade da atenção primária à saúde se destaca o PCATool (Primary Care Assessment Tool) - desenvolvido por Starfield e colaboradores na Universidade de John Hopkins (JHU) para avaliar o alcance e extensão dos atributos (acesso de primeiro contato do indivíduo com o sistema de saúde, longitudinalidade, integralidade e coordenação da atenção; orientação familiar, orientação comunitária e competência cultural) da atenção primária (4).

As avaliações da qualidade em saúde tornamse cada vez mais essencial na medida em que auxiliam gestores e profissionais da saúde para a tomada de decisão, possibilitem capacitá-los nas decisões referentes às intervenções necessárias que atendam às necessidades de saúde da população através de uma maior eficiência, efetividade dos serviços e contribua para a melhoria da qualidade do cuidado em saúde (5).

O presente estudo teve por objetivo avaliar os atributos da APS, sob a percepção de usuários de um município em um Estado da região Norte do Brasil através da ferramenta do PCATool.

\section{MÉTOdos}

Este artigo constitui-se numa pesquisa avaliativa com abordagem quantitativa e delineamento transversal realizada com usuários da atenção primária distribuídos em cinco Distritos de Saúde (Disa Norte, Disa Sul, Disa Oeste, Disa Leste e Disa Rural) no Município de Manaus (AM) cuja população estimada em 2018 era de 2.145.444 habitantes (6). Manaus conta com 190 equipes ativas de Estratégia Saúde da Família (97 de ESF; 87 ESF com Saúde Bucal 
Modalidade - C/ SB- I; 03 ESF RIBEIRINHAS C/ SB; e 03 ESF FLUVIAIS C/SB) em 214 Unidades Básicas de Saúde (UBS) distribuídas nos distritos, e cobertura populacional de ESF de $30,23 \%$, enquanto as unidades tradicionais de saúde respondem por $44,35 \%$ dessa população Foram abordados, apenas os usuários dos Distritos da área urbana de Manaus em função da dificuldade e custo de locomoção para a área Rural de Manaus. (7)

A população do estudo constituiu-se por residentes e pessoas atendidas nos distritos de saúde pelas equipes de ESF. Foram convidados a participar da pesquisa, os usuários adultos maiores de 18 anos por meio da amostragem aleatória simples. Como critério de exclusão adotou-se não apresentar condições físicas e/ou mentais para responder ao questionário. $\mathrm{O}$ dimensionamento amostral dos entrevistados foi realizado através de um cálculo de amostra estratificada nos distritos de saúde selecionados na pesquisa. Definiu-se, então, uma população finita e um erro absoluto de $5 \%$. O resultado do dimensionamento demonstrou a necessidade da utilização de 384 participantes, contudo, a amostra final totalizou em 395 adultos (112 no Distrito Norte; 91 no Distrito Sul; 97 no Distrito Leste e, 95 no Distrito Oeste).

As entrevistas foram realizadas em sua maioria no interior das UBS, no horário de atendimento aos usuários, e estes eram convidados a participar da pesquisa, com explicações sobre os objetivos, riscos e benefícios da sua participação, ressaltando-se o anonimato. Inicialmente foi aplicado com os usuários um questionário do perfil sociodemográfico com questões relativas ao sexo, idade, anos de estudo e situação conjugal dos participantes, número de filhos, renda familiar, acesso a plano privado de saúde, ocupação, motivo principal para a busca do serviço da UBS e frequência com que procura a UBS. Os participantes assinaram o Termo de Consentimento Livre e Esclarecido, em conformidade com a legislação vigente.

Os dados foram coletados entre outubro de 2018 a março de 2019 por intermédio da aplicação do instrumento Primary Care Assessment Tool (PCATool-Brasil) - usuários adultos. A versão validada do PCATool do Adulto contém 87 itens divididos em 10 componentes relacionados aos atributos da APS. As respostas são apresentadas do tipo Likert, com intervalo de um a quatro (1 a 4) para cada atributo. As respostas possíveis para cada um dos itens foram: "com certeza sim" (valor=4), "provavelmente sim" (valor=3), "provavelmente não" (valor=2), "com certeza não" (valor=1) e "não sei/não lembro" (valor=9). Os escores para cada um dos atributos ou seus componentes são calculados pela média aritmética simples dos valores das respostas dos itens que compõe cada atributo ou seu componente. O resultado geral da avaliação pelo PCATool é calculado a partir de duas medidas: o Escore Essencial, que é a média dos escores dos componentes que pertencem aos atributos essenciais, e o Escore Geral, que é a média dos escores dos componentes dos atributos essenciais, acrescida dos escores dos atributos derivados. Esses resultados caracterizam o grau de orientação do serviço ou do sistema de saúde aos atributos da Atenção Primária à Saúde. (8).

Após a consolidação dos dados relativos a cada atributo, os valores são transformados em escala contínua, variando entre zero e dez, utilizando a fórmula a seguir: Escala $=[$ (escore obtido -1$) \times 10] / 3$. Valores de escores $\geq 6,6$ foram definidos como elevados e equivalentes ao valor três ou mais ( $\geq 3$ ) na escala Likert, enquanto valores $\leq 6,6$ foram considerados 
baixos (9). Foram calculados os Escores Essencial e Geral da atenção primária no município analisado.

As respostas foram organizadas em um banco de dados criado no software Microsoft Excel for Windows. Foram calculadas as estatísticas descritivas dos atributos da atenção primária: frequências absolutas e relativas, médias, desvio-padrão.

O presente artigo apresenta parte dos resultados de pesquisa aprovada pelo CEP da COEP-UFMG - Comissão de Ética em Pesquisa da UFMG sob o No. 2.876.275, de 06 de setembro de 2018, que também aprovou o Termo de Consentimento Esclarecido que foi assinado por todos os entrevistados.

\section{Resultados e Discussões}

Neste estudo, foram realizadas 395 entrevistas com usuários da ESF do município de Manaus. As características sociodemográficas dos participantes da pesquisa encontram-se na Tabela 1.

O perfil dos usuários que frequentam as unidades de saúde da família é constituído de mulheres (90,89\%); em idade fértil (idade entre 25 e 59$)$; pardas $(81,52 \%)$; donas de casa $(41,52 \%)$; casadas $(63,04 \%)$ e com filhos (até quatro filhos, $73,92 \%$ ); que frequentam a UBS pelo menos uma vez ao mês (31,65\%); para fazer, principalmente, o seu acompanhamento de rotina médica (83,04\%). São usuários de baixa renda, pois (50\%) percebem até um salário mínimo para atender suas necessidades básicas de sobrevivência; quase $40 \%$ desses têm apenas o ensino médio completo, e $26,33 \%$ não possuíam o ensino fundamental completo. Tais características corroboram com outros estudos da área, que destacam as mulheres como grandes usuárias desse sistema, uma vez que elas são as principais responsáveis por levarem seus filhos, e demais parentes para receberem os serviços e ações de atenção primária (10) (11) (12).

É uma população que depende basicamente do SUS, pois, não possui plano privado de saúde (92,91\%), em conformidade com estudo anterior que buscou analisar a evolução do perfil de utilização de serviços de saúde, entre 2003 e 2008, no Brasil e nas suas macrorregiões, a partir do banco de dados da PNAD - Pesquisa Nacional por Amostras de Domicílios. (13) (14)

A tabela 2 apresenta os escores médios, desvio-padrão, valores máximos e mínimos dos atributos da atenção primária avaliados pelos usuários manauaras. Nota-se que dois atributos essenciais (Acessibilidade e Integralidade) e um atributo derivado (Orientação Comunitária) foram avaliados com baixo valor no seu escore médio, inferior a 6,6 que é considerada a referência do ponto de corte para a avaliação de uma atenção primária de alta qualidade. Esses valores encontrados se assemelham com alguns estudos sobre o tema do acesso aos serviços de saúde na atenção primária. (15)

No questionário do PCATool, o atributo acesso de primeiro contato possui duas subdimensões avaliadas: acessibilidade e utilização. A acessibilidade busca avaliar, por exemplo, se é fácil para o usuário conseguir atendimento clínico no mesmo dia em caso de adoecimento; ou se ele pode se aconselhar com a equipe de profissionais por telefone se precisar; investiga ainda, quais os horários e dias de atendimento da equipe de ESF nas unidades de saúde. Verifica-se, portanto, um entrave à acessibilidade nesse quesito para os usuários nas unidades de saúde da estratégia da saúde, uma vez que as referidas unidades funcionam em horário comercial, e somente 
nos dias úteis da semana. Não há disponibilidade de atendimento para o usuário no turno noturno, o que limita o acesso aos trabalhadores em geral. Por outro lado, percebe-se pouca disposição das equipes de ESF, em utilizar formas comunicativas com os usuários baseadas nas atuais tecnologias, o que reforça a necessidade do usuário em se deslocar até a UBS para sanar suas dúvidas, obter informações sobre algum procedimento clínico, medicação, etc. (16)

Com relação ao atributo da integralidade do cuidado à saúde, no PCATool, este atributo pretende captar a oferta de serviços disponíveis e prestados pelo serviço de atenção primária. No tocante ao atributo Integralidade - serviços disponíveis - este recebeu baixo valor no seu escore médio $(5,92)$ pelos usuários, sendo que os itens avaliados nesse atributo contem perguntas sobre procedimentos considerados cirúrgicos e invasivos ( $G 10$ Sutura de um corte; que necessite de pontos; G 14 colocação de tala; G15 remoção de verrugas), os quais em sua maioria não são realizados pelas ESF.

A subdimensão - integralidade serviços prestados recebeu um valor baixo no escore médio $(4,79)$ em linha com outros estudos de avaliação da atenção primária com ênfase na integralidade na atenção primária. (17) Nesse atributo os subitens: H3 - aconselhamento sobre o uso de cinto de segurança ou assentos seguros para crianças ao andar de carro e H9 se o usuário tem uma arma de fogo e é orientado a como guardá-la com segurança foram mal avaliados pelos comunitários. Outrossim, a maioria das UBS da família em Manaus estão inseridas nas chamadas "áreas vermelhas", ou seja, em áreas de bairros com grande incidência de crimes relacionados com o tráfico de drogas e outros crimes. Portanto, discutir sobre a guarda segura de armas não é conveniente e pode expor o profissional a constrangimentos e riscos. (18) (19)

A Orientação Comunitária é um atributo no qual o escore médio apurado foi baixo, principalmente nos itens $\mathrm{J4}$, que questiona aos usuários se os profissionais das equipes de ESF realizam pesquisas para aferir se os serviços oferecidos estão atendendo às necessidades das pessoas; o J15 avalia se as ESF realizam pesquisas na comunidade para identificar os problemas de saúde que ela deveria conhecer e o J6, indaga a respeito se o usuário ou outro membro de sua família participa do Conselho Local de Saúde; todos esses itens foram negativamente avaliados, configurando assim, um índice de insatisfação por parte dos usuários nesses atributos. Tais características negativas relacionadas a esse atributo também foram destacadas em outros estudos. (20) (21)

A ESF é formada por uma equipe multiprofissional que tem por objetivo realizar ações e serviços de saúde no âmbito da atenção primária para uma determinada comunidade. $\mathrm{Na}$ sua prática de trabalho cotidiana, a equipe realiza o mapeamento do território sob sua responsabilidade, a partir de pesquisas na comunidade para identificação de problemas e situações de riscos de saúde que são relevantes e recorrentes naquela realidade social, cultural, demográfica e epidemiológica das famílias. Com o conhecimento adquirido junto à população é possível fazer um planejamento adequado para as intervenções de ações de saúde naquela comunidade. Portanto, a avaliação negativa do atributo orientação comunitária, além de mostrar a insatisfação dos usuários, mostra uma realidade onde os preceitos originais da ESF estão enfraquecidos. (22)

Os demais atributos analisados longitudinalidade, coordenação de cuidados e 
orientação familiar foram bem avaliados pelos usuários da APS em Manaus, assim como em outros estudos utilizando o PCATool para avaliação da Atenção Primária à Saúde. De fato, uma maior a convivência das equipes da ESF com a família, sugere um conhecimento melhor de suas demandas e problemas econômicos sociais e, possibilita a criação de vínculos de confiança entre a equipe e os usuários. (24) (25)

Em que pese o atributo da Coordenação de Cuidados ter obtido um escore médio positivo na pesquisa, há uma pergunta discriminada neste atributo na subdimensão - integração de cuidados, item E4, o qual questiona ao usuário se foi "discutido" com ele os diferentes serviços aonde ele poderia realizar um atendimento com um médico ou serviço especializado. Esse item dificilmente tem avaliação positiva pelos usuários, uma vez que, não é dado o direito ao usuário escolher, por exemplo, realizar um serviço especializado, um exame de próstata, uma consulta com um oftalmologista perto da sua residência. A marcação desse tipo de exame e consultas médicas especializadas é realizada através de um sistema regulador (SISREG), que avalia segundo critérios técnicos, entre eles, a disponibilidade de vagas e atendimento. Logo, é muito comum, o usuário quando consegue a consulta com o especialista ele ser deslocado para um lugar bem distante da sua moradia, 0 que lhe causa extrema insatisfação com o sistema que regula essa distribuição de atendimentos a especialistas e serviços especializados em Manaus.

Não obstante, feito os cálculos necessários para apurar o escore Essencial, este alcançou a média de 7,16 variando entre 1,75 e 8,67; e o escore Geral atingiu 7,02 variando de 1,81 a 8,77 , ambos alcançaram a média acima de 6,6, configurando uma alta avaliação da atenção primária pelos usuários manauaras para o período analisado.

\section{CONSIDERAÇÕES FINAIS}

O instrumento PCATool - Brasil é uma importante ferramenta criada para avaliar a qualidade dos serviços na atenção primária à saúde, de acordo com itens que procuram representar os atributos (as características) encontrados em sistemas de saúde evidências de alta qualidade, relacionando aqueles atributos com aspectos estruturais e de processos nos serviços de saúde.

O perfil sociodemográfico dos usuários do ESF no Município de Manaus é composto por mulheres, em idade fértil, pardas, donas de casa, casadas e com filhos, que frequentam a UBS pelo menos uma vez ao mês, para atendimento de rotina e não possuem plano privado de saúde. Trata-se de uma população altamente dependente do SUS, portanto, as politicas de saúde no campo da atenção primária tem um peso enorme nos resultados de saúde dessa população.

O atributo acesso de primeiro contato na subdimensão acessibilidade apresentou baixo valor em seu escore médio em Manaus, sugerindo, portanto que o serviço de saúde possui uma insuficiente acessibilidade, em particular, no que tange aos horários de atendimento e formas de comunicação entre a equipe e a população atendida nas UBS da família.

No tocante ao baixo valor médio do escore do atributo integralidade na percepção dos usuários da pesquisa, percebe-se que o atributo citado está aquém das necessidades de saúde da população atendida pelas equipes de Estratégia saúde na Família, uma vez que não estão sendo oferecidas ações integrais de 


\section{APS em Revista}

Vol. 1, n. 2, p. 98/111 | Maio/Agosto - 2019

ISSN 2596-3317 - DOI 10.14295/aps.v1i2.27

Sá, L. Y. B. A. V.

promoção e prevenção de saúde nos cuidados dos adultos de forma satisfatória e efetiva.

O atributo Orientação Comunitária teve uma avaliação negativa por parte dos usuários na pesquisa, sugerindo que as equipes de ESF realizam poucas pesquisas para conhecer 0 perfil epidemiológico, econômico, social e cultural da comunidade com vistas ao atendimento das necessidades da saúde daquela. Tampouco estimula a participação da comunidade dessas últimas.

Neste estudo, o Escore Geral da APS, calculado pela média dos atributos essencial e derivado, mostrou forte orientação à APS na perspectiva dos usuários em relação às ações e serviços de saúde ofertados pelas equipes de ESF no município de Manaus (AM).

\section{REFERÊNCIAS BIBLIOGRÁFICAS}

(1) STARFIELD, B. Atenção Primária: equilíbrio entre necessidades de saúde, serviços e tecnologia. Brasília: UNESCO, Ministério da Saúde, 2002. 726 p.

(2) SENNA, M. C. M.; SANTOS, M. A. ATENÇÃO PRIMÁRIA À SAÚDE NA AGENDA PÚBLICA BRASILEIRA: dilemas entre focalização e universalidade. Revista de Políticas Públicas. v. 21, n. 1 (2017) p.403-424.

(3) FELISBERTO, Eronildo et al. Política de monitoramento e avaliação da atenção básica no Brasil de 2003 a 2006: contextualizando sua implantação e efeitos. Rev. Bras. Saude Mater. Infant., Recife, v.9, n.3, p.339-357, Set. 2009. Disponível em http://www.scielo.br/scielo.php?script=sci_arttext \&pid=S1519-

38292009000300013\&lng=en\&nrm=iso>. Acesso em: 09. Mai.2019.

(4) SHI L, STARFIELD B, XU J. Validating the adult primary care assessment tool. J Fam Pract 2001; 50(2):161-175.
(5) TANAKA, Oswaldo Yoshimi; TAMAKI, Edson Mamoru. O papel da avaliação para a tomada de decisão na gestão de serviços de saúde. Ciênc. saúde coletiva, Rio de Janeiro, v. 17, n. 4, p. 821828, Apr. 2012. Available from <http://www.scielo.br/scielo.php?script=sci_arttext \&pid=S141381232012000400002\&lng=en\&nrm=iso $>$ access on $08 \quad$ May 2019. http://dx.doi.org/10.1590/S1413-

81232012000400002 .

(6) INSTITUTO BRASILEIRO DE GEOGRAFIA E ESTATÍSTICA (IBGE). ESTIMATIVAS DA POPULAÇÃO RESIDENTE PARA OS MUNICÍPIOS E PARA AS UNIDADES DA FEDERAÇÃO COM DATA DE REFERÊNCIA EM 10 DE JULHO DE 2018. Rio de Janeiro: IBGE, 2018. Disponível em: https://biblioteca.ibge.gov.br/visualizacao/livros/liv 101609.pdf . Acesso em: 08. Maio. 2019.

(7) BRASIL. Ministério da Saúde. Histórico de Cobertura da Saúde da Família no Brasil [internet]. Brasília, DF: MS; 2017 [acesso em 2019 mar 21]. Disponível

em: http://dab.saude.gov.br/portaldab/historico_cobert ura_sf.php

(8) BRASIL. Ministério da Saúde. Secretaria de Atenção em Saúde. Departamento de Atenção Básica. Manual do instrumento de avaliação da atenção primária à saúde: primary care assessment tool PCATool - Brasília: Ministério da Saúde, 2010. 80 p.: il. - (Série A. Normas e Manuais Técnicos)

(9) HARZHEIM, Erno et al. Validação do instrumento de avaliação da atenção primária à saúde: PCAToolBrasil adultos. Revista Brasileira de Medicina de Família e Comunidade, [S.I.], v. 8, n. 29, p. 274-284, out. 2013. ISSN 2179-7994. Disponível em: <https://www.rbmfc.org.br/rbmfc/article/view/829 /588>. Acesso em: 08 maio 2019. doi:https://doi.org/10.5712/rbmfc8(29)829.

(10) IBAÑEZ N, ROCHA JSY, CASTRO PC, RIBEIRO MCSA, FORSTER AC, NOVAES MHD, et al. Avaliação do desempenho da atenção básica no estado de São Paulo. Ciência \& Saúde Coletiva. 2006 Jul-Set; 11(3): 683-703. 


\section{APS em Revista}

Vol. 1, n. 2, p. 98/111 | Maio/Agosto - 2019

ISSN 2596-3317-DOI 10.14295/aps.v1i2.27

Sá, L. Y. B. A. V.

(11) GUIBU IA, MORAESJC, GUERRA JUNIOR AA, COSTA EA, ACURCIO FA, COSTA KS, et al. Características principais dos usuários dos serviços de atenção primária à saúde no Brasil. Rev Saude Publica 2017;51 Supl 2:17s.

(12) PEREIRA, Aline Braido et al. Perfil social do usuário do sistema único de saúde na atenção primária em saúde. Revista de Epidemiologia e Controle de Infecção, Santa Cruz do Sul, v. 2, n. 3, p. 94-98, jul. 2012. ISSN 2238-3360. Disponível em: <https://online.unisc.br/seer/index.php/epidemiolo gia/article/view/2746/2191>. Acesso em: 08 maio 2019. doi:https://doi.org/10.17058/reci.v2i3.2746.

(13) VIACAVA, Francisco; BELLIDO, Jaime G. Condições de saúde, acesso a serviços e fontes de pagamento, segundo inquéritos domiciliares. Ciênc. saúde coletiva, Rio de Janeiro, v. 21, n. 2, p. 351370, Feb. 2016. Available from <http://www.scielo.br/scielo.php?script=sci_arttext \&pid=S1413-

$81232016000200351 \&$ lng=en\&nrm=iso $>$. access on 08 May 2019. http://dx.doi.org/10.1590/141381232015212.19422015.

(14) SILVA, Z. P. et al. Perfil sociodemográfico e padrão de utilização dos serviços de saúde do Sistema Único de Saúde (SUS), 2003-2008. Ciência \& Saúde Coletiva, Rio de Janeiro, v. 16, n. 9, p. 38073816, 2011.

(15) TESSER, Charles Dalcanale, NORMAN, Armando Henrique e VIDAL, Tiago Barra. Acesso ao cuidado na Atenção Primária à Saúde brasileira: situação, problemas e estratégias de superação. Saúde em Debate [online]. 2018, v. 42, n. spe1 [Acessado 8 Maio 2019],pp.361-378.Disponível em: $<$ https://doi.org/10.1590/0103-

11042018 S125>.ISSN

2358-2898. https://doi.org/10.1590/0103-11042018S125.

(16) CHOMATAS, Eliane et al. Avaliação da presença e extensão dos atributos da atenção primária em Curitiba. Revista Brasileira de Medicina de Família e Comunidade, [S.I.], v. 8, n. 29, p. 294-303, out. 2013. ISSN 2179-7994. Disponível em: <https://www.rbmfc.org.br/rbmfc/article/view/828 >. Acesso em: 09 maio 2019. doi:https://doi.org/10.5712/rbmfc8(29)828.
(17) PAULA FA. Avaliação dos atributos da atenção primária na saúde do adulto na Estratégia de Saúde da Família de Diamantina/MG. Diamantina (MG): Universidade Federal dos Vales do Jequitinhonha e Mucuri; 2013.

(18) CÉSAR, Cíntia Cristina Mariano. Os desafios da promoção da saúde em territórios conflagrados e de extrema vulnerabilidade social: um olhar a partir do Complexo da Maré. 2016, 105 f. Dissertação (Mestrado Profissional em Educação Profissional em Saúde) - Fundação Oswaldo Cruz. Escola Politécnica de Saúde Joaquim Venâncio, Rio de Janeiro, 2016.

(19) DINIZ SGM, Damasceno SS, Coutinho SED, Toso BRGO, Collet N. Avaliação do atributo integralidade na atenção à saúde da criança. Rev Gaúcha Enferm. 2016 dez; 37(4):e57067. doi: http://dx.doi.org/10,1590/19831447.2016.04.57067.

(20) ALENCAR, Monyk Neves de et al. Avaliação do enfoque familiar e orientação para a comunidade na Estratégia Saúde da Família. Ciênc. saúde coletiva, Rio de Janeiro, v. 19, n. 2, p. 353-364, Feb. 2014 Available from <http://www.scielo.br/scielo.php?script=sci_arttext \&pid=S1413-

$81232014000200353 \& \operatorname{lng}=e n \& n r m=i s o>$. access on 08 May 2019. http://dx.doi.org/10.1590/141381232014192.08522012. access on 08 May 2019. http://dx.doi.org/10.1590/0102-311X00132114.

(21) BATISTA VCL, Ribeiro LCC, Ribeiro CDAL, Paula FA, Araújo A. Avaliação dos atributos da atenção primária à saúde segundo os profissionais de saúde da família. SANARE. 2016; 15(2):87-93.

(22) VAN STRALEN, Cornelis Johannes et al. Percepção dos usuários e profissionais de saúde sobre atenção básica: comparação entre unidades com e sem saúde da família na Região Centro-Oeste do Brasil. Cad. Saúde Pública, Rio de Janeiro, v. 24, supl. 1, p. s148-s158, 2008. Available from $<$ http://www.scielo.br/scielo.php?script=sci_arttext \&pid=S0102-

$311 X 2008001300019 \&$ Ing=en\&nrm=iso $>$. access on 09 May 2019. http://dx.doi.org/10.1590/S0102311X2008001300019. 


\section{ABSTRACT}

Background: This article aims to evaluate the attributes of primary health care from the perspective of users of the public health system in the city of Manaus (AM).

Methods: A cross-sectional, descriptive study was carried out with a population of $\mathbf{3 9 5}$ registered users and attended by the Family Health Strategy teams in the basic health units, which answered with the use of the PCATool-Brazil instrument (adults version) and a sociodemographic questionnaire.

Results: The attributes of accessibility, completeness, and community orientation obtained averages of low scores. The Overall Score, measured by the Mean of the Essential Score and the Derived Score, surpassed the cut bridge of 6.6. The mean of the Essential Score was 7.16.

Conclusions: Health services and actions in Manaus in general have a high degree of orientation towards Primary Health Care, although two essential attributes and a derived attribute have indicators that need to be improved.

Keywords: Health Assessment; Assessment of Primary Care; Primary Health Care. 


\section{APS em Revista}

Vol. 1, n. 2, p. 98/111 | Maio/Agosto - 2019

ISSN 2596-3317-DOI 10.14295/aps.v1i2.27

Sá, L. Y. B. A. V.

Quadro 1. Instrumento Primary Care Assessment Tool (PCATool-Brasil) - usuários adultos

\begin{tabular}{|c|c|c|}
\hline \multicolumn{3}{|c|}{ PCATOOL - BRASIL - VERSÃO ADULTO } \\
\hline \multicolumn{3}{|c|}{ ATRIBUTOS ESSENCIAIS E DERIVADOS DA APS } \\
\hline $\begin{array}{l}\text { ACESSO DE PRIMEIRO CONTATO } \\
\text { ACESSIBILIDADE }\end{array}$ & $\begin{array}{l}\text { No atributo essencial designado por } \\
\text { acesso de primeiro contato do indivíduo } \\
\text { com o sistema de saúde busca-se avaliar a } \\
\text { acessibilidade e utilização do serviço de } \\
\text { saúde como fonte de cuidado a cada novo } \\
\text { problema ou novo episódio de um mesmo } \\
\text { problema de saúde, com exceção das } \\
\text { verdadeiras emergências e urgências } \\
\text { médicas. }\end{array}$ & $\begin{array}{l}\text { 1. Grau de Afiliação com } \\
\text { Serviço de Saúde (A). } \\
\text { Constituído por } 3 \text { itens (A1, A2 } \\
\text { e A3) } \\
\text { 2. Acesso de Primeiro Contato } \\
\text { - Utilização (B). Constituído } \\
\text { por } 3 \text { itens (B1, B2 e B3). } \\
\text { 3. Acesso de Primeiro Contato } \\
\text { - Acessibilidade (C). } \\
\text { Constituído por } 12 \text { itens (C1, } \\
\text { C2, C3, C4, C5, C6, C7, C8, C9, } \\
\text { C10, C11 e C12). }\end{array}$ \\
\hline LONGITUDINALIDADE & $\begin{array}{l}\text { Longitudinalidade refere-se à existência } \\
\text { de uma fonte continuada de atenção, } \\
\text { assim como sua utilização ao longo do } \\
\text { tempo. }\end{array}$ & $\begin{array}{l}\text { 4.Longitudinalidade (D). } \\
\text { Constituída por } 14 \text { itens (D1, } \\
\text { D2, D3, D4, D5, D6, D7, D8, D9, } \\
\text { D10, D11, D12, D13 e D14). }\end{array}$ \\
\hline COORDENAÇÃO DA ATENÇÃO & $\begin{array}{l}\text { Por Coordenação da atenção subentende- } \\
\text { se que "o provedor de atenção primária } \\
\text { deve ser capaz de integrar todo cuidado } \\
\text { que o paciente recebe através da } \\
\text { coordenação entre os serviços". }\end{array}$ & $\begin{array}{l}\text { 5. Coordenação - Integração } \\
\text { de Cuidados (E). Constituído } \\
\text { por } 8 \text { itens (E2, E3, E4, E5, E6, } \\
\text { E7, E8 e E9). } \\
\text { 6. Coordenação - Sistema de } \\
\text { Informações (F). Constituído } \\
\text { por } 3 \text { itens (F1, F2 e F3). }\end{array}$ \\
\hline INTEGRALIDADE & $\begin{array}{l}\text { O atributo da Integralidade pretende } \\
\text { captar a oferta de serviços disponíveis e } \\
\text { prestados pelo serviço de atenção } \\
\text { primária. Isto é, compreende todas as } \\
\text { ações de atenção e cuidado de saúde } \\
\text { disponibilizada ao usuário, inclusive, } \\
\text { aquelas cuja necessidade gera } \\
\text { encaminhamentos para especialidades } \\
\text { médicas focais, hospitais, entre outros. }\end{array}$ & $\begin{array}{l}\text { 7. Integralidade - Serviços } \\
\text { Disponíveis (G). Constituído } \\
\text { por } 22 \text { itens (G1, G2, G3, G4, } \\
\text { G5, G6, G7, G8, G9, G10, G11, } \\
\text { G12, G13, G14, G15, G16, G17, } \\
\text { G18, G19, G20, G21, G22) } \\
\text { 8. Integralidade - Serviços } \\
\text { Prestados (H). Constituído por } \\
\text { 13 itens para mulheres (H1, } \\
\text { H2, H3, H4, H5, H6, H7, H8, H9, } \\
\text { H10, H11, H12, H13) e } 11 \text { itens } \\
\text { para homens (H1, H2, H3, H4, }\end{array}$ \\
\hline
\end{tabular}




\section{APS em Revista}

Vol. 1, n. 2, p. 98/111 | Maio/Agosto - 2019

ISSN 2596-3317-DOI 10.14295/aps.v1i2.27

Sá, L. Y. B. A. V.

\begin{tabular}{|c|l|l|}
\hline & & $\mathrm{H} 5, \mathrm{H6}, \mathrm{H} 7, \mathrm{H} 8, \mathrm{H9}, \mathrm{H} 10, \mathrm{H} 11)$. \\
\hline ORIENTAÇÃO FAMILIAR & $\begin{array}{l}\text { Os serviços de atenção primária devem } \\
\text { centrar-se na família, para bem avaliar } \\
\text { como responder às necessidades de saúde } \\
\text { de seus membros. }\end{array}$ & $\begin{array}{l}\text { 9. Orientação Familiar (I). } \\
\text { Constituído por } 3 \text { itens (I1, I2, } \\
\text { I3) }\end{array}$ \\
\hline ORIENTAÇÃO COMUNITÁRIA & $\begin{array}{l}\text { Os serviços de atenção primária devem } \\
\text { estar orientados para a comunidade, } \\
\text { conhecendo suas necessidades de saúde. }\end{array}$ & $\begin{array}{l}\text { 10. Orientação Comunitária (J) } \\
\text { Jonstituída por } 6 \text { itens (J1, }\end{array}$ \\
\hline JOM, J4, J5, J6).
\end{tabular}

Fonte: STARFIELD et al ( 2001); BRASIL (2010)

Tabela 1 - Perfil sociodemográfico dos usuários da Estratégia da Saúde da Família. Manaus, AM, Brasil, 2019

\begin{tabular}{|c|c|c|}
\hline \multirow[t]{2}{*}{ Variável } & \multicolumn{2}{|l|}{ Manaus } \\
\hline & $N(=395)$ & $\%$ \\
\hline \multicolumn{3}{|l|}{ Sexo } \\
\hline Masculino & 36 & 9,11 \\
\hline Feminino & 359 & 90,89 \\
\hline \multicolumn{3}{|l|}{ Faixa etária } \\
\hline 18 a 24 & 45 & 11,39 \\
\hline 25 a 40 & 134 & 33,92 \\
\hline 41 a 59 & 154 & 38,99 \\
\hline 60 ou mais & 60 & 15,19 \\
\hline não informado & 2 & 0,51 \\
\hline \multicolumn{3}{|l|}{ Cor/raça } \\
\hline Negra & 19 & 4,81 \\
\hline Branca & 51 & 12,91 \\
\hline Amarela & 2 & 0,51 \\
\hline Parda & 322 & 81,52 \\
\hline Indígena & 1 & 0,25 \\
\hline
\end{tabular}

\section{Status civil}


APS em Revista

Vol. 1, n. 2, p. 98/111 | Maio/Agosto - 2019

ISSN 2596-3317-DOI 10.14295/aps.v1i2.27

Sá, L. Y. B. A. V.

\begin{tabular}{lcc}
\hline Casado(a)/ Ajuntado(a) & 249 & 63,04 \\
\hline Separado(a)/ Divorciado(a) & 32 & 8,10 \\
\hline Solteiro(a) & 23 & 23,04 \\
\hline Viúvo(a) & & 5,82 \\
\hline Número de filhos & 44 & 11,14 \\
\hline Nenhum & 171 & 43,29 \\
\hline de 1 a 2 & 121 & 30,63 \\
\hline de 3 a 4 & 38 & 9,62 \\
\hline de 5 a 6 & 10 & 2,53 \\
\hline de 7 a 8 & 7 & 1,77 \\
\hline de 9 a 10 & 1 & 0,25 \\
\hline de 11 a 12 & 3 & 0,76 \\
\hline não informado & & \\
\hline
\end{tabular}

\section{Continuação}

\section{Escolaridade}

\begin{tabular}{lcc}
\hline Analfabeto & 4 & 1,01 \\
\hline Fundamental Incompleto & 104 & 26,33 \\
\hline Fundamental Completo & 16 & 4,05 \\
\hline Ens. Médio Incompleto & 47 & 11,90 \\
\hline Ens. Médio Completo & 157 & 39,75 \\
\hline Superior Incompleto & 26 & 6,58 \\
\hline Superior Completo & 37 & 9,37 \\
\hline Outro & 3 & 0,76 \\
\hline Não informado & 1 & 0,25
\end{tabular}

\section{Possui plano de saúde privado?}

\begin{tabular}{lcc}
\hline Sim & 27 & 6,84 \\
\hline Não & 367 & 92,91 \\
\hline não informado & 1 & 0,25 \\
\hline Ocupação & & 36,20 \\
\hline Trabalha & 143 & 36 \\
\hline
\end{tabular}




\section{APS em Revista}

Vol. 1, n. 2, p. 98/111 | Maio/Agosto - 2019

ISSN 2596-3317 - DOI 10.14295/aps.v1i2.27

Sá, L. Y. B. A. V.

\begin{tabular}{lcc}
\hline Dona de casa & 164 & 41,52 \\
\hline Desempregado(a) & 54 & 13,67 \\
\hline Aposentado(a) & 20 & 5,06 \\
\hline Pensionista & 2 & 0,51 \\
\hline não informado & 12 & 3,04
\end{tabular}

\section{Renda mensal (em salário mínimo-sm)}

\begin{tabular}{lcc}
\hline até $1 \mathrm{sm}$ & 199 & 50,38 \\
\hline de 1,01 a $2 \mathrm{sm}$ & 135 & 34,18 \\
\hline de 2,01 a $3 \mathrm{sm}$ & 24 & 6,08 \\
\hline mais de $3 \mathrm{sm}$ & 6 & 1,52 \\
\hline não informado & 31 & 7,85
\end{tabular}

Motivo principal para a busca do serviço da UBS

\begin{tabular}{llc}
\hline Tratamento odontológico & 10 & 2,53 \\
\hline Consulta de rotina (Check-up) & 328 & 83,04 \\
\hline Atendimento de urgência & 3 & 0,76 \\
\hline Realização de exames laboratoriais & 3 & 0,76 \\
\hline Marcação de consultas ou exames especializados & 17 & 4,30 \\
\hline Outro & 33 & 8,35 \\
\hline não informado & 1 & 0,25 \\
\hline Frequência com que procura a UBS & 125 & 31,65 \\
\hline Mensal & 33 & 8,35 \\
\hline A cada 2 meses & 67 & 16,96 \\
\hline a cada 3 meses & 73 & 18,48 \\
\hline A cada 6 meses & 46 & 11,65 \\
\hline Anualmente & 50 & 12,66 \\
\hline Esporadicamente & 1 & 0,25 \\
\hline não informado & & \\
\hline
\end{tabular}

Fonte: Elaboração própria 


\section{APS em Revista}

Vol. 1, n. 2, p. 98/111 | Maio/Agosto - 2019

ISSN 2596-3317 - DOI 10.14295/aps.v1i2.27

Sá, L. Y. B. A. V.

Tabela 2 - Valores médios e respectivos desvios padrões dos atributos da Atenção Primária à Saúde conferidos pelos usuários adultos na avaliação, Manaus, (AM), 2019

\begin{tabular}{|c|c|c|c|c|}
\hline Atributos da APS & Média por escore & Desvio Padrão & Máximo & Mínimo \\
\hline$A$ & 9,46 & 2,01 & 10,00 & 0,00 \\
\hline B & 8,57 & 2,36 & 10,00 & 0,00 \\
\hline C & 3,76 & 1,26 & 7,50 & 0,00 \\
\hline $\mathrm{D}$ & 8,34 & 1,14 & 10,00 & 4,29 \\
\hline$E$ & 8,99 & 1,31 & 10,00 & 0,00 \\
\hline $\mathrm{F}$ & 8,32 & 2,09 & 10,00 & 0,00 \\
\hline G & 5,92 & 1,60 & 8,64 & 0,91 \\
\hline $\mathrm{H}$ & 4,79 & 1,98 & 9,39 & 0,00 \\
\hline 1 & 7,55 & 2,86 & 10,00 & 0,00 \\
\hline J & 5,36 & 2,36 & 10,00 & 0,00 \\
\hline Essencial & 7,16 & 1,12 & 8,67 & 1,75 \\
\hline Geral & 7,02 & 1,18 & 8,77 & 1,81 \\
\hline
\end{tabular}

Fonte: Elaboração própria 